\title{
Laserpointer-Interaktion für große, hochauflösende Displays
}

\author{
Werner A. König, Hans-Joachim Bieg, Harald Reiterer \\ Arbeitsgruppe Mensch-Computer Interaktion, Universität Konstanz
}

\section{Zusammenfassung}

Aufgrund des limitierten Sehvermögens des Menschen muss sich ein Anwender vor großen, hochauflösenden Displays frei bewegen können, um kleinste Details pixelgenau wahr zu nehmen oder einen Überblick über die gesamte Darstellungsfläche von mehreren Quadratmetern zu erhalten. Im Gegensatz zu konventionellen Eingabegeräten wie Maus und Tastatur beschränken Laserpointer den Anwender nicht in seiner Bewegungsfreiheit, sondern ermöglichen unabhängig von der Position zum Display eine sehr intuitive und direkte Art der Interaktion. In diesem Beitrag wird eine Interaktionsbibliothek vorgestellt, welche im Hinblick auf Präzision und verzögerungsfreier Steuerung erstmals auch den Einsatz von Laserpointer-Tracking bei großen, hochauflösenden Displays ermöglicht. In einem Vergleichsexperiment wurde die Interaktionsbibliothek in Kombination mit einem Infrarotlaserpointer gegenüber einer klassischen Maus als Standardeingabegerät bei unterschiedlichen Distanzen evaluiert. Der signifikante Performancevorteil der Maus von 12,5\% scheint in Anbetracht der gewonnenen Bewegungsfreiheit und der unmittelbaren Interaktionsweise mit dem Laserpointer eher gering ins Gewicht $\mathrm{zu}$ fallen. Im Vergleich zu bisherigen Systemen konnte der Rückstand des Laserpointers um über 50\% reduziert werden, was größtenteils auf die geringe Bewegungslatenz, das präzise Tracking und die wirksame Kompensation des Zitterns der Hand zurückzuführen ist. Ferner wurde bei der Studie ein signifikanter Distanzeffekt beim Laserpointer hinsichtlich Performance und Fehlerrate festgestellt.

\section{$1 \quad$ Motivation}

Zur Analyse und Exploration von komplexen Informationsräumen werden in den unterschiedlichsten wissenschaftlichen und industriellen Bereichen vermehrt große, hochauflösende Displays (LHRD) als Ausgabegeräte verwendet. Diese zeichnen sich typischerweise durch eine Bildschirmdiagonale von mehr als fünf Metern und eine Auflösung von über acht Megapixel aus. Aufgrund der hohen Auflösung können nicht nur sehr große Datenmengen gleichzeitig dargestellt werden, sondern implizit auch Detail- und Übersichtsansicht in einer Abbildung vereint werden. Dem Anwender bietet sich aus größerem Abstand zum Display

Konstanzer Online-Publikations-System (KOPS)

URL: http://www.ub.uni-konstanz.de/kops/volltexte/2007/3138/

URN: http://nbn-resolving.de/urn:nbn:de:bsz:352-opus-31381 
eine gute Übersicht über die gesamte Darstellungsfläche und somit auch über die visualisierten Informationen, wobei die Details aufgrund des limitierten menschlichen Sehvermögens nur bedingt oder gar nicht wahrzunehmen sind. Um auch diese sehen zu können, muss der Betrachter sich notwendigerweise dem Display nähern. Beim Herantreten an das Display, was auch als physisches Zooming bezeichnet werden könnte, erhält der Anwender demgemäß immer mehr Detailinformationen, muss sich aber auf einen Teilbereich des Displays fokussieren und verliert damit die Übersicht über das ganze Display. Um dieser Art der physischen Navigation gerecht zu werden, muss ein Eingabegerät für LHRDs eine ausreichend große Bewegungsflexibilität bieten. Traditionelle Eingabegeräte wie Tastatur und Maus schränken dieses Benutzerverhalten durch die Notwendigkeit einer stationären Auflagefläche ein und ermöglichen nur eine sehr indirekte Art der Interaktion. Mehr Flexibilität versprechen kabellose Mäuse mit eingebautem Rotationssensor oder Präsentationshelfer mit integriertem Joystick, welche alternativ zur klassischen Maus als relative Zeigeinstrumente eingesetzt werden können. Diese weisen aber nicht nur erheblich niedrigere Performancewerte im Vergleich zur traditionellen Maus auf (MacKenzie \& Jusoh 2001), sondern eignen sich auch nur bedingt für handschriftliche Annotationen und Skizzierungen.

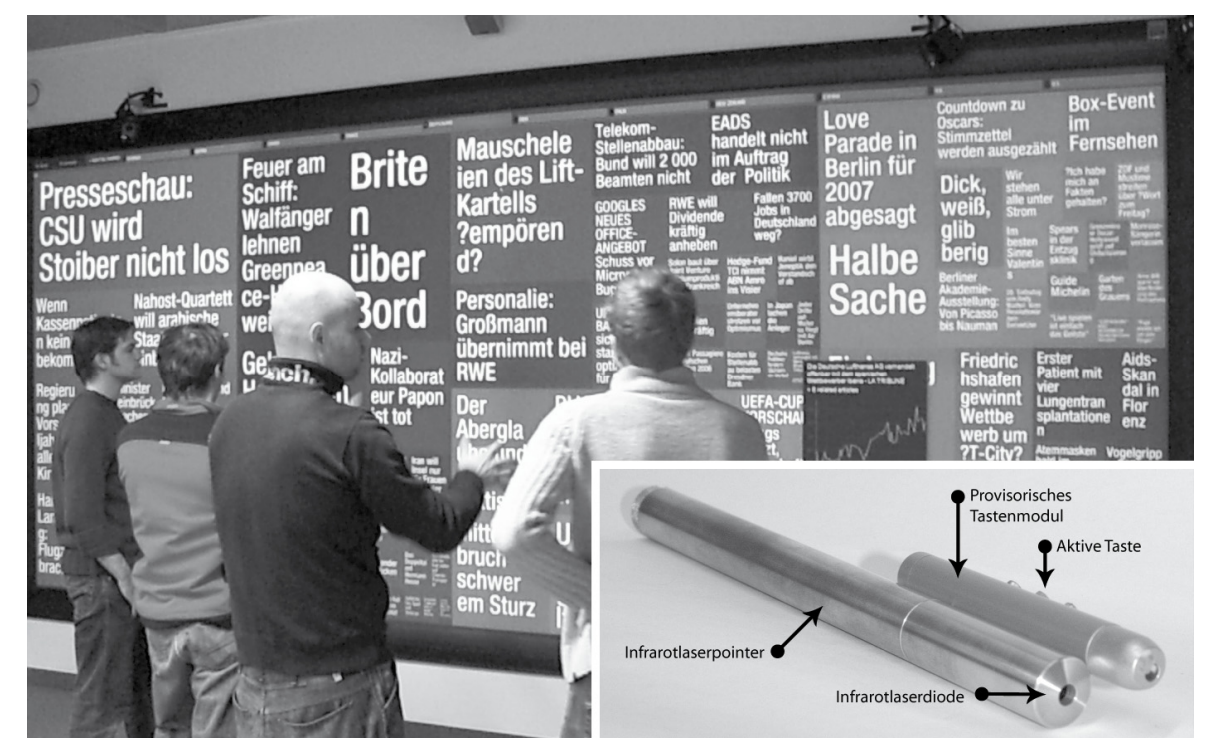

Abbildung 1: Newsmap-Visualisierung auf der Konstanzer Powerwall und evaluierter Infrarotlaserpointer

Ein größeres Potential als direktes, flexibles und vor allem intuitives Eingabegerät für große, hochauflösende Displays sehen wir im Laserpointer-Tracking. Hierbei wird der schon als Zeigeinstrument für Präsentationen übliche Laserpointer auch für die Positionierung des Cursors oder generell zur Steuerung der Bedienungsoberfläche verwendet. Der Laserpointer zeichnet sich dabei durch seine intuitive Handhabung als Verlängerung der Hand und damit durch eine sehr direkte Interaktion aus. Der Bewegung eines Laserpointers als absolutes Eingabegerät kann der Anwender unmittelbar eine gleichgeartete, simultane Bewegung des Cursors zuordnen, wohingegen bei der traditionellen Maus die Bewegung in zwei unterschiedlichen Ebenen (horizontale Auflagefläche vs. vertikales Display) stattfindet. Die für 
das Benutzerverhalten vor LHRDs notwendige Mobilität ist bei Laserpointern per se gegeben, da nicht die Position des Gerätes, sondern die Reflexion des gebündelten Laserstrahls auf der Darstellungsfläche für die Eingabe verwendet wird. Folglich kann der Anwender direkt vor einem Display bzw. sogar auf einem Display schreiben oder auch noch aus mehreren Metern Entfernung mit dem identischen Gerät auf identische Weise interagieren. Bisherige Laserpointer-Systeme wurden hauptsächlich für klassische Präsentationsszenarien mit einem Projektor oder für Demonstrationen an Mehrprojektorsystemen entwickelt. LHRDs wie die Konstanzer Powerwall mit 5,20 x 2,15 m Darstellungsfläche und einer Auflösung von 4640 x 1920 Pixeln stellen jedoch ganz neue Herausforderung an die generelle Laserpointer-Interaktion und speziell an die Präzision und Geschwindigkeit des TrackingVerfahrens. Im Rahmen dieses Beitrags wird erstmals eine flexible und skalierbare Interaktionsbibliothek vorgestellt, welche eine sehr direkte und nahezu verzögerungsfreie Eingabe mittels Laserpointer-Tracking auch für große, hochauflösende Displays ermöglicht. Die Präzision wird dabei nicht nur durch subpixel-genaue Erkennungsverfahren und die Anbindung von theoretisch beliebig vielen Kameras erhöht, sondern auch durch eine wirksame Kompensation des natürlichen Zitterns der Hand mittels eines angepassten Kalman-Filters. Des Weiteren wird ein Laserpointer mit infraroter Laserdiode vorgestellt, welcher eine Eingabe ohne sichtbare Reflexionen auf dem Display ermöglicht und sich aufgrund der geringen Strahlungsintensität (Laserschutzklasse 1) völlig unbedenklich einsetzen lässt. Die Interaktionsbibliothek mit Infrarotlaserpointer wurde in einer Evaluationsstudie auf Basis des ISO Standards 9241-9 an der Konstanzer Powerwall mit dem bisherigen Standardeingabegerät, einer konventionellen Maus, verglichen. Bevor in Kapitel 3 die Interaktionsbibliothek und in Kapitel 4 die Evaluation näher betrachtet werden, sollen im Folgenden zuerst relevante Arbeiten und Evaluationsstudien aufgeführt werden.

\section{$2 \quad$ Verwandte Arbeiten}

Preiswerte Laserpointer dienen schon seit Jahren einer breiten Anwendergruppe bei Vorlesungen, Vorträgen und Präsentationen als optischer Zeigestock. Die Idee zur konsequenten Fortführung der Benutzung des Laserpointers vom passiven Zeigeinstrument zum aktiven Eingabegerät entstand schon Ende der 90-er Jahre und wurde in zahlreichen prototypischen Systemen und Evaluationen bis heute weiterverfolgt.

Ein der ersten technischen Vorarbeiten leisteten dabei Kirstein und Müller (1998), welche ein System zum Erkennen von einzelnen Laserpunkten auf einer Projektionsfläche mithilfe einer Videokamera vorstellten. Bereits bei einer verhältnismäßig geringen Erkennungsrate von 50\% und einer Abtastrate von lediglich 20 Bildern pro Sekunde (fps) erwies sich dabei der Ansatz grundsätzlich als vielversprechend. Als Interaktion konnte mit dem Laser nicht nur die Mausbewegung emuliert werden, sondern das Verharren auf einem Punkt (Dwelling) löste einen Button-Press-Event und das Abschalten des Lasers ein Button-Release-Event aus. Wissen et al. (2001) wandten Laserpointer-Tracking auf Mehrprojektorsysteme mit einer Darstellungsfläche von 6,5 x 1,6 m mittels eines Kameraverbunds von drei Kameras an. Diese nahmen jeweils einen Teilbereich des Displays auf und waren über ein Netzwerk mit 
einem gemeinsamen Bildrechner verbunden. Einen ähnlichen Ansatz wählten Chen und Davis (2001) für ihr hochauflösendes Rückprojektionsdisplay „Interactive Mural“ mit 1,8 x 0,6 m und einer Auflösung von 3796 x 1436 Pixel. Acht Kameras konnten mit 60 Hz Zwischenzeilenabtastung gleich mehrere Laserpunkte parallel lokalisieren und vereinten die einzelnen Abschnitte der Bewegungspfade mithilfe separater Kalman-Filter. Ahlborn et al. (2005) legten ebenfalls den Schwerpunkt auf die technischen Aspekte eines skalierbaren Trackingsystems mit einer robusten und effizienten Punkterkennung mittels verschiedenster Optimierungsalgorithmen. Matveyev und Göbel (2003) stellten einen speziellen Infrarotlaserpointer vor, dessen Lichtstrahl in drei Teile mit einem vordefinierten Winkel geteilt wurde. Eine Kamera ermittelte so nicht nur die Position, sondern konnte aus der Anordnung und den Abständen zwischen den Punkten auf die Distanz zwischen Anwender und Projektionswand schließen. Ebenso konnten durch die Manipulation der Strahlwinkel im Laserpointer verschiedene Aktionen visuell dem System übermittelt werden.

Zusätzlich zu den technischen Vorarbeiten wurden in unterschiedlichsten Studien Laserpointer-Systeme mit traditionellen Eingabegeräten wie Maus oder Smartboards verglichen und Parameter ermittelt, welche auf die Performance des Laserpointers Einfluss nahmen. Cavens et al. verglichen 2002 eine klassische Maus mit einem rot leuchtenden Laserpointer und in einer weiteren Studie letzteren mit einem Infrarotlaserpointer. Hierbei wiesen Maus und roter Laserpointer ähnlich gute Bewegungszeiten auf, wohingegen der Infrarotlaserpointer signifikant schlechter abschnitt. Peck (2001) untersuchte relevante Parameter für das Design von Laserpointer-Interaktionstechniken und fand in einer Studie mit 10 Probanden heraus, dass diese zwischen 0,9 und 1,4 Sekunden benötigten, um nach dem Einschalten des Lasers auf ein bestimmtes Ziel zu zeigen. Darüber hinaus musste für eine zuverlässige Erkennung einer Dwelling-Aktion mindestens nochmals eine weitere Sekunde der Laser in einem Bereich gehalten werden. Myers et al. (2002) verglichen mittels eines Tapping-Tests eine traditionelle Maus mit einem Laserpointer und einem berührungssensitivem Smartboard. Erwartungsgemäß schnitt die direkte Interaktion auf dem Smartboard am besten mit einem Durchsatz von 11,80 Bits/s (siehe Kapitel 4.3), gefolgt von der Maus mit 6,98 Bits/s und dem Laserpointer mit 5,08 Bits/s ab. Auf ein ähnliches Ergebnis kamen Oh und Stürzlinger (2002) bei einem Vergleich Maus versus Laserpointer mit einem Durchsatz von 3,98 Bits/s zu 3,04 Bits/s. Aufgrund unterschiedlicher Berechnungen des Durchsatzes sind die absoluten Zahlen hier nicht direkt vergleichbar, es kann jedoch in allen Fällen eine um mindestens 30\% höhere Performance der Maus im Vergleich zum Laserpointer festgestellt werden.

Die bisherigen Studien wurden an konventionellen Projektionsdisplays (1,8 x 1,2 m, Oh \& Stürzlinger 2002), mit dementsprechend mittleren Amplituden (Distanz zwischen Zielen: 60 cm, Myers et al. 2002) und geringem Abstand zum Display (1,5 m, Myers et al. 2002) durchgeführt. Fraglich ist dabei, ob die ermittelten Werte auch auf große, hochauflösende Displays wie der Konstanzer Powerwall mit 5,20 x 2,15 m Darstellungsfläche und einer Auflösung von 4640 x 1920 Pixeln übertragbar sind. Hier können Objekte über fünf Meter voneinander entfernt sein und der Benutzer muss je nach Distanz zum Display nicht nur die Hand, sondern auch den Kopf drehen, um das Ziel treffen bzw. überhaupt sehen zu können. In Kapitel 4 soll die Frage der Übertragbarkeit und generellen Eignung von LaserpointerInteraktion erstmals auch für große, hochauflösende Displays durch eine Evaluationsstudie beantwortet werden. Vorher wird im Kapitel 3 das evaluierte System näher betrachtet. 
Obwohl es in den letzten Jahren eine Reihe an wissenschaftlichen Arbeiten und Umsetzungen bezüglich Laserpointer-Tracking gab, so fehlte bisher ein Referenzsystem, welches die Teillösungen der einzelnen Arbeiten innerhalb einer flexiblen und dennoch stabilen Interaktionsbibliothek vereint. Zusätzlich zu diesem hat die hier vorgestellte Bibliothek auch den Anspruch speziell für die Interaktion mit großen, hochauflösenden Displays geeignet zu sein. Außer der um ein vielfaches größeren Darstellungsfläche bei LHRDs, muss auch der hohen Auflösung des Displays und der Flexibilität des Anwenders Rechnung getragen werden. Die Interaktionsbibliothek erlaubt durch die Anbindung einer beliebigen Anzahl von Kameras eine genügend hohe Präzision bei der Erkennung des Laserpunktes. Die Interaktionspräzision wird ferner durch einen dynamischen Kalman-Filter erhöht, welcher das natürlich Zittern der menschlichen Hand durch eine Kombination von unabhängigen Modellen für langsame und schnelle Bewegungen und separate Vorhersagen für horizontale und vertikale Koordinaten kompensieren soll. Eine direkte Steuerung ohne merkliche Bewegungslatenz soll dem Anwender durch den Einsatz von Industriekameras mit Abtastraten von über 80 fps und entsprechend schneller Bildverarbeitung und Signalfilterung ermöglicht werden. Dabei können die Kameras vor oder hinter dem Display, zentral oder schräg, von oben oder unten, flexibel angeordnet werden. Eine automatische Kalibrierung ermittelt dementsprechend zusätzlich zu den intrinsischen Kameraparametern (radiale und tangentiale Verzerrung) auch die extrinsischen, welche sich aus der Positionierung zum Display ergeben. Eine weitere Eigenheit dieser Interaktionsbibliothek ist die Unterstützung von sichtbaren (z.B. rot, grün, blau) und infraroten Laserstrahlen. Bisherige Systeme arbeiteten meist mit roten Laserpointern, wobei auf dem Display entweder nur der rote Laserpunkt oder dieser zusätzlich zum virtuellen Mauscursor zu sehen ist. Die Tatsache, dass infolge von Verzögerungen und Ungenauigkeiten beim Tracking Laserpunkt und Mauscursor sich weder immer gleich verhalten, noch genau übereinander liegen, kann zu Irritationen aufseiten des Anwenders führen. Infrarote Laserpointer ermöglichen das alleinige Anzeigen des virtuellen Mauscursors, welcher dementsprechend je nach Systemstatus in seiner Erscheinung beliebig variiert werden kann und sich somit erwartungskonform verhält. Ferner ist beim sichtbaren Laser das Zittern des Anwenders - beispielsweise in einer Präsentationssituation - für jeden deutlich sichtbar. Beim infraroten Laserpointer wird das Zittern durch den Kalman-Filter reduziert, wodurch der virtuelle Cursor sich auch noch bei höherem Stresslevel weitestgehend stabil verhält.

\subsection{Hardwareumgebung}

Grundsätzlich ist die Interaktionsbibliothek hardwareunabhängig bezüglich Display, Kamera und Laserpointer ausgelegt. Es werden sowohl Einzel- als auch Mehrfachprojektionen beliebiger Größe und Auflösung unterstützt. Für die nachfolgende Studie und als Referenzumgebung wurde die Konstanzer Powerwall verwendet - ein Rückprojektionsdisplay, welches mittels acht Hochleistungsprojektoren betrieben wird und durch ein homogenes Gesamtbild besticht. Für die Bildaufnahme können prinzipiell übliche Web- oder Videokameras per USB oder Firewire angeschlossen werden. Eine präzise und nahezu verzögerungsfreie Interaktion 
wird jedoch erst durch Spezialkameras mit hoher Auflösung und Abtastrate ermöglicht. Die Bedeutung einer möglichst geringen Verzögerung im Trackingprozess unterstrichen MacKenzie und Ware (1993), welche eine direkte Korrelation zwischen dem Durchsatz des jeweiligen Eingabegerätes und der Interaktionslatenz, also der Gesamtverzögerung beim Tracking festgestellt haben. Hierbei stellte sich aber auch heraus, dass unterhalb einer Verzögerung von $25 \mathrm{~ms}$ der negative Einfluss auf die Performance vernachlässigbar gering wird. Ausgehend von einer durchschnittlichen Verzögerung von 8,3 ms infolge der Darstellungsrate $(60 \mathrm{~Hz})$ und einer maximalen Berechnungs- und Übertragungszeit von $10 \mathrm{~ms}$ für die Bildanalyse, sollte aus der Abtastrate der Kamera nicht mehr als eine Verzögerung von 6,7 ms im Mittel resultieren, um insgesamt unterhalb den $25 \mathrm{~ms}$ Gesamtverzögerung $(8,3+10+6,7=25 \mathrm{~ms}) \mathrm{zu}$ bleiben. Um dieser Anforderung gerecht zu werden, wurden für die Interaktionsbibliothek drei identische Industriekameras (IDS 1540-C, ca. 650€) mit 80 fps (6,25 ms gemittelte Verzögerung) bei 640 x 512 Pixel Auflösung und einem 4,8 mm Weitwinkelobjektiv ausgewählt. Diese wurden vertikal zentriert hinter der Powerwall angebracht und nehmen jeweils 1/3 der Darstellungsfläche auf. Aufgrund der Client-Server-Architektur der Interaktionsbibliothek können die Kameras an verschiedenen Rechnern angeschlossen und die Bildanalyse verteilt ausgeführt werden, woraus eine höhere Flexibilität und Skalierbarkeit resultiert. Prinzipiell werden von der Interaktionsbibliothek Laserpointer unterschiedlichster Wellenlängen und Intensitäten als Eingabegeräte unterstützt. Der hier verwendete Laserpointer ähnelt in seiner Form einem Standardlaserpointer, beinhaltet jedoch eine Laserdiode der Laserklasse 1, welche mit $0,55 \mathrm{~mW}$ Infrarotlicht der Wellenlänge $785 \mathrm{~nm}$ emittiert. Dank der geringen Strahlungsintensität ist dieser Infrarotlaser, im Gegensatz zu den sonst oft verwendeten rot leuchtenden Laserpointern der Schutzklasse 3, für den Menschen absolut ungefährlich und kann bedenkenlos eingesetzt werden. Aufgrund von fehlenden Buttons, wurden zur Emulation von Maustasten für die Vergleichsstudie auf das Gehäuse des Infrarotlaserpointers die Buttons (Page up/down) eines normalen Präsentationslasers montiert (siehe Abbildung 1). Es ist geplant, in einer weiteren Designiteration die Buttons direkt in das Gehäuse zu integrieren und deren Statusänderungen über Funk zu übertragen.

\subsection{Tracking-Verfahren}

Aufgrund der Vielfalt an möglichen Konfigurationen bezüglich Display, Kamera und deren Positionierung wird einmalig vor dem eigentlichen Tracking eine Kalibrierung des Systems vorgenommen. Hierbei werden automatisch die intrinsischen Parameter der Kamera, die Aufstellung zum Display und der jeweilige Aufnahmebereich ermittelt bzw. festgelegt. Ebenso werden störende Reflexionen von Projektoren, Lampen oder Sonneneinstrahlung registriert und im weiteren Verlauf jeweils aus dem aktuellen Bild heraus gerechnet. Für das eigentliche Tracking wird die Belichtungszeit der Kamera verringert, wodurch das intensitätsschwache Umgebungslicht sich nur noch leicht abbildet und der wesentlich hellere Laserpunkt klar herauskommt. Bei der Verwendung des infraroten Laserpunkts wird ein optischer Infrarotfilter an die Kamera angebracht, welcher nur noch Licht über $750 \mathrm{~nm}$ Wellenlänge durchlässt und damit das sichtbare Licht weitestgehend herausfiltert. Daher können selbst noch sehr schwache Infrarotdioden der Laserschutzklasse 1 stabil erkannt werden. Für die eigentliche Punkterkennung, wird das RGB-Bild in ein Grauwertbild konvertiert, wobei 
jeweils der hellste Farbwert pro Pixel übernommen und eventuell noch verstärkt wird. Anschließend werden helle Regionen identifiziert und angelehnt an Oh und Stürzlinger (2002) deren Zentrum ermittelt. Hierbei wird ein nach Intensitätswerten gewichtetes Zentrum berechnet, dessen Genauigkeit nicht durch die Kameraauflösung limitiert ist. Trotz einer „subpixel“" genauen Lokalisation des Laserpunktes fällt es - infolge des natürlichen Zitterns der menschlichen Hand - dem Anwender schwer einen Punkt präzise auf dem Display zu treffen und ebenfalls auch auf diesem stabil zu verweilen. In einer Studie von Peck (2001) stellte sich eine durchschnittliche Abweichung von ca. $0,4^{\circ}$ beim Verharren auf einem Zielpunkt über einen Zeitraum von drei Sekunden heraus. Hochgerechnet auf die Konstanzer Powerwall würde der Anwender bei $3 \mathrm{~m}$ Entfernung schwerlich Objekte treffen können, welche kleiner als 18 Pixel $(2,09 \mathrm{~cm})$ in Höhe und Breite messen. Gerade aber bei großen, hochauflösenden Displays, deren Hautpanwendung die Visualisierung von komplexen Informationsräumen darstellt, beeinträchtigt diese Ungenauigkeit stark den Benutzer in seiner Interaktion. Zur Kompensation dieser Problematik wendet die hier vorgestellte Interaktionsbibliothek einen Kalman-Filter an, welcher auf Basis der bisher gemessenen Koordinaten, ermittelten Abweichungen und der Bewegungsgeschwindigkeit eine Vorhersage für die nächste Position des Laserpunktes trifft, diese anhand der Realdaten korrigiert und iterativ die Bewegungsmodellierung anpasst. Dabei wird sowohl technisch als auch menschlich bedingtes Rauschen in den Koordinatendaten reduziert. Um sowohl schnelle Bewegungen als auch präzises Verharren zu unterstützen, wird nach dem Multi-Modell-Ansatz je nach Güte der Vorhersage entweder ein statisches oder ein dynamisches System oder eine gewichtete Kombination beider für die Vorhersage ausgewählt. Bei der letztendlichen Positionierung beispielsweise eines virtuellen Mauscursors werden dementsprechend nicht die tatsächlich gemessenen Koordinaten, sondern die geglättete Vorhersagen genutzt, wodurch erst stabiles Halten einer Position und weiche Bewegungen ermöglicht werden.

\section{$4 \quad$ Experiment}

Um die Eignung der Interaktionsbibliothek für LHRDs zu überprüfen, wurde ein Experiment auf Basis der ISO 9241-9 an der Konstanzer Powerwall durchgeführt. Primäres Ziel war es, das Laserpointersystem mit dem Standardeingabegerät Maus zu vergleichen. Existierende Studien legen nahe, dass mit der Maus präziser und schneller interagiert werden kann. Darüber hinaus sollte auch der Einfluss der Distanz zum Display auf die Performance der Geräte untersucht werden. Studien von Peck (2001) und Myers et al. (2002) betrachteten die Abweichung beim Zielen auf einen fixen Punkt über mehrere Distanzen zum Display. Die Ergebnisse beider Studien unterstreichen die intuitive Annahme, dass sich die Performance des Laserpointers, bedingt durch den Effekt des Zitterns der Hand, mit zunehmender Distanz verringert. Grundlage des durchgeführten Experiments bildete die unidirektionale Tappingaufgabe (Fitts' Tapping Tasks) wie sie in ISO 9241-9 skizziert wird. Bei der Aufgabe werden zwei rechteckige Ziele mit einer gewissen Mitte-zu-Mitte Distanz A (Amplitude) und Breite W (Width) präsentiert (siehe Abbildung 2). Die Testperson soll die Ziele nacheinander so schnell und präzise wie möglich selektieren. Diese Art des Tests wurde in zahlreichen Studien zur Evaluation von Eingabegeräten genutzt (vgl. Soukoreff \& MacKenzie 2004). 


\subsection{Teilnehmer und experimentelles Design}

Die Evaluation wurde mit 16 Teilnehmern als 2 x 2 Within-Subjects Design mit den Faktoren Gerät (Laserpointer, Maus) und Distanz (3 m, 6 m) angelegt. Um Lern- und Ermüdungseffekte auszugleichen, wurden die VP zufällig vier Gruppen zugeordnet, bei denen die Reihenfolge der Faktorlevel nach dem Latin Square Schema festgelegt wurde. Als abhängige Maße wurden die Bewegungszeit (MT), Fehlerquote (ER) sowie der effektive Durchsatz $\left(\mathrm{IP}_{\mathrm{e}}\right)$ ermittelt. Hierbei kommt dem effektiven Durchsatz eine besondere Rolle zu, da dieser sowohl Präzision als auch Schnelligkeit der Interaktion in sich vereint. Die Berechnungen erfolgten anhand der Vorgaben der ISO 9241-9 bzw. analog zu Soukoreff \& MacKenzie (2004). Bei einem Durchlauf wurde die Breite W (80 px, 140 px entspr. ca. $9 \mathrm{~cm}, 15 \mathrm{~cm})$ sowie die Mitte-zu-Mitte Distanz A (550 px, 1350 px, 3800 px entspr. ca. $62 \mathrm{~cm}, 151 \mathrm{~cm}$, $426 \mathrm{~cm}$ ) variiert, sodass sich Schwierigkeitswerte zwischen 2,3 Bits (einfach) und 5,6 Bits (schwer) ergaben und die Ziele im zentralen, mittleren und peripheren Bereich der Powerwall zu sehen waren. $\mathrm{Zu}$ jeder der 2 x 3 Konfigurationen führten die Versuchspersonen 15 Trials (entspricht 16 Klicks) durch. Die Abfolge der W-A Konfigurationen war stets zufällig.
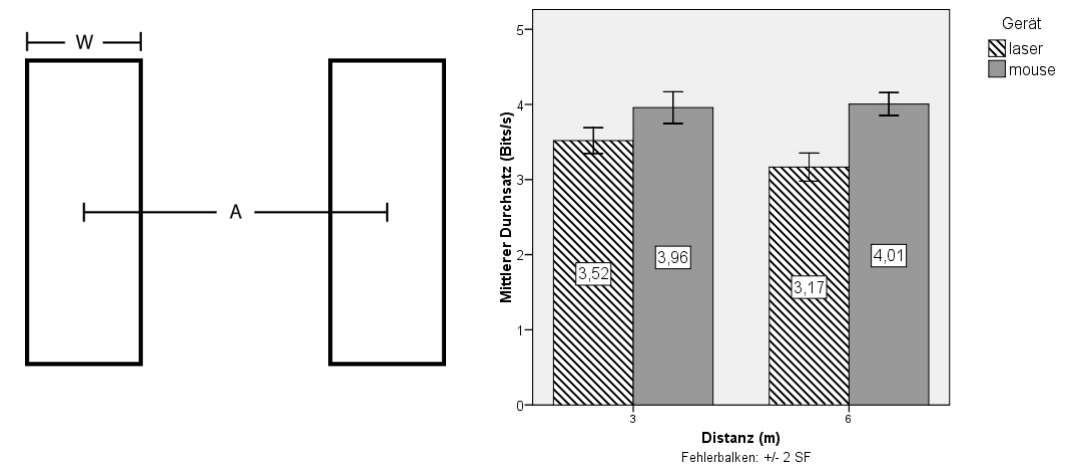

Abbildung 2: Unidirektionale Tappingaufgabe (links), durchschnittlicher effektiver Durchsatz (rechts)

\subsection{Versuchsaufbau \& Ablauf}

Mit beiden Geräten sollten die Probanden vor der 5,20 x 2,15 Meter großen Powerwall im Stehen interagieren. Als Auflagefläche für die Maus wurde dazu ein Pult (Höhe ca. 1,10 m) verwendet. Zur Aufzeichnung der Interaktion und zur Darstellung der Ziele wurde eine für diese Evaluation entwickelte Software eingesetzt, die mit der maximalen Displayauflösung von 4640 x 1920 Pixeln betrieben wurde. Als Mauszeiger wurde der standardmäßig eingestellte Zeiger von MS Windows Server 2003 verwendet. Zu Beginn des Experiments erhielten die VP eine Erläuterung zur Vorgehensweise. Dabei wurden die VP angewiesen die Ziele so schnell und präzise wie möglich zu selektieren. Die Teilnehmer wurden gebeten sich mittig vor das Display der Powerwall an entsprechende Markierungen zu stellen. Bei jeder der nun folgenden vier Geräte-Distanz Kombinationen wurde den VP die Möglichkeit gegeben, sich vor dem eigentlichen Test an Gerät und Distanz zu gewöhnen. Dazu wurde fünf Mal hintereinander eine nicht zur Auswertung herangezogene Tappingaufgabe mit je 65 Klicks durchgeführt. Über alle Faktorstufen hinweg führte jede VP insgesamt 1664 Klicks durch. 


\subsection{Ergebnisse}

Von den insgesamt aufgezeichneten 5760 Trials wurden 18 nicht zur Auswertung herangezogen, da diese auf untypische Interaktionen wie beispielsweise unabsichtliche Doppelklicks zurückgeführt werden konnten. Wie erwartet schnitt die Maus hinsichtlich des effektiven Durchsatzes $\left(\mathrm{IP}_{\mathrm{e}}\right.$ ) besser ab als der Laserpointer (siehe Abbildung 2). Bei einer Distanz von $3 \mathrm{~m}$ ergab sich für den Laserpointer ein mittlerer $\mathrm{IP}_{\mathrm{e}}$ Wert von 3,52 Bits/s und 3,96 Bits/s für die Maus. Bei $3 \mathrm{~m}$ betrug die Fehlerrate des Laserpointers 15\%, die der Maus $8 \%$. Im Durchschnitt wurde bei $3 \mathrm{~m}$ eine Bewegungszeit von $1086 \mathrm{~ms}$ für den Laserpointer und $954 \mathrm{~ms}$ für die Maus gemessen. Bei der Varianzanalyse zeigten sich ein signifikanter Effekt für den Faktor Gerät über alle Maße: $\operatorname{IP}_{e}\left(F_{1,15}=30,570 ; p<0,001\right)$, Err $\left(F_{1,15}=57,767 ; p<0,001\right)$ und MT $\left(\mathrm{F}_{1,15}=21,487 ; \mathrm{p}<0,001\right)$. Im Gegensatz zur Maus wurde erwartet, dass sich die Performance des Laserpointers mit zunehmender Distanz verschlechtert. Die Ergebnisse konnten diese Vermutung bestätigen. Signifikante Distanz-Gerät Interaktionseffekte konnten für $\mathrm{IP}_{\mathrm{e}}\left(\mathrm{F}_{1,15}=8,627 \mathrm{p}=0,010\right)$ und $\operatorname{Err}\left(\mathrm{F}_{1,15}=6,489, \mathrm{p}=0,022\right)$ festgestellt werden. Die Analyse des einfachen Haupteffekts der Distanz zeigte, dass der IP $\mathrm{P}_{\mathrm{e}}$ Wert des Laserpointers bei einer Distanz von $3 \mathrm{~m}$ (3,52 Bits/s) signifikant $\left(\mathrm{F}_{1,15}=11,59\right.$; $\left.\mathrm{p}=0,004\right)$ höher war als bei $6 \mathrm{~m}$ (3,17 Bits/s) wohingegen bei der Maus kein signifikanter Unterschied festgestellt werden konnte. Zudem verschlechterte sich die Genauigkeit mit der Probanden den Laserpointer bedienen konnten signifikant $\left(\mathrm{F}_{1,15}=19,76 ; \mathrm{p}<0,001\right)$ von $15 \%$ bei $3 \mathrm{~m}$ auf $20 \%$ bei $6 \mathrm{~m}$. Wiederum konnte für die Maus kein signifikanter Unterschied festgestellt werden. Zwar erhöhte sich beim Laserpointer auch die Bewegungszeit von $1086 \mathrm{~ms}(3 \mathrm{~m})$ auf $1133 \mathrm{~ms}$ (6 m), dieser Unterschied ist jedoch nicht signifikant.

\section{$5 \quad$ Schlussfolgerung}

Im Rahmen dieses Beitrags wurde eine auf Laserpointer-Tracking basierende Interaktionsbibliothek vorgestellt, welche im Gegensatz zu bisherigen Systemen speziell auch den Anforderungen von großen, hochauflösenden Displays in Bezug auf Präzision, Skalierbarkeit und Effizienz genügen soll. Um die Eignung des Systems für LHRDs festzustellen, wurde die Interaktionsbibliothek in Kombination mit einem Infrarotlaserpointer in einem Vergleichsexperiment gegenüber der Maus als Standardeingabegerät an der Konstanzer Powerwall (5,20 x 2,15 m, 4640 x 1920 Pixeln) evaluiert. Die Ergebnisse zeigen, dass nach wie vor ein Performancevorteil von ca. 12,5\% (IP, $3 \mathrm{~m}$ ) für die Maus besteht, dieser aber im Vergleich zu bisherigen Studien trotz größerer Distanz um mehr als 50\% reduziert werden konnte (siehe Kapitel 2). Größtenteils kann diese Verbesserung durch die geringe Bewegungslatenz sowie die hohe Trackingpräzision in Kombination mit dem Multi-Modell Kalman-Filter begründet werden. Dem Performancevorteil der Maus steht die gewonnene Flexibilität durch die Unabhängigkeit des Laserpointers von einer stationären Auflagefläche gegenüber. Diese Flexibilität spielt insbesondere bei LHRDs eine entscheidende Rolle, da hier je nach Position zum Display entweder die ganze Darstellungsfläche überblickt oder die Details wahrgenommen werden können und somit der Benutzer dazu gezwungen ist, sich ständig vor dem 
Display zu bewegen. Die Veränderung der Genauigkeit des Laserpointers bei größerer Distanz legt es nahe, Interaktionsziele entsprechend der Distanz zum Display zu skalieren, um einen konstanten Performancelevel zu gewährleisten. Zudem könnte eine variable Anpassung des Kalman-Filters zur jeweiligen Distanz und der damit verbundenen Ungenauigkeit infolge des natürlichen Zitterns der Hand die Distanzeffekte reduzieren.

\section{$6 \quad$ Literaturverzeichnis}

Ahlborn, B. A.; Thompson, D.; Kreylos, O.; Hamann, B.; Staadt, O. G. (2005): A practical system for laser pointer interaction on large displays. In: Proc. VRST'05, ACM Press, New York, 106-109.

Cavens, D.; Vogt, F.; Fels, S.; Meitner, M. (2002): Interacting with the big screen: pointers to ponder. In: Proc. CHI 2002 Extended Abstracts, ACM Press, New York, 678-679.

Chen, X.; Davis, J. (2001): LumiPoint: Multi-User Laser-Based Interaction on Large Tiled Displays. Technical Report, Stanford University.

Kirstein, C.; Müller, H. (1998): Interaction with a Projection Screen Using a Camera-tracked Laser Pointer. In: Proc. MMM'98, IEEE Computer Society, Washington, 191-192.

MacKenzie, I. S.; Jusoh, S. (2001): An evaluation of two input devices for remote pointing. In: Proc. EHCI'01, Springer-Verlag, Heidelberg, 235-249.

MacKenzie, I. S.; Ware, C. (1993): Lag as a determinant of human performance in interactive systems. In: Proc. CHI'93, ACM Press, New York, 488-493.

Matveyev, S. V; Göbel, M. (2003): The optical tweezers: multiple-point interaction technique. In: Proc. VRST '03, ACM Press, New York, 184-187.

Myers, B. A.; Bhatnagar, R.; Nichols, J.; Peck, C. H.; Kong, D.; Miller, R.; Long, A. C. (2002): Interacting at a distance: measuring the performance of laser pointers and other devices. In: Proc. $\mathrm{CHI}$ '02, ACM Press, New York, 33-40.

Oh, J.; Stürzlinger W. (2002): Laser pointers as collaborative pointing devices. In: Proc. Graphics Interface 2002, 141-149.

Peck, C. H. (2001): Useful parameters for the design of laser pointer interaction techniques. In: Proc. CHI '01, ACM Press, New York, 461-462.

Soukoreff, R. W.; MacKenzie, I. S. (2004): Towards a standard for pointing device evaluation, perspectives on 27 years of Fitts' law research in HCI. In: IJHCS, 61(6), 751-789.

Wissen, M.; Wischy, M. A.; Ziegler, J. (2001): Realisierung einer laserbasierten Interaktionstechnik für Projektionswände. Mensch \& Computer 2001, Teubner Verlag, Stuttgart, 135-143.

\section{Danksagung}

Die hier vorgestellte Forschungsarbeit entstand im Kontext des Forschungsverbundes „Interaktive Visualisierung für Gigapixel Displays" im Rahmen des Förderprogramms Informationstechnik Baden-Württemberg (BW-FIT). Für Anregungen, Ideen und Mitarbeit danken wir Vladimir Bondarenko, Anton Stasche, Toni Schmidt, Jens Gerken und Sebastian Rexhausen. 